\title{
ROTATIONAL ANISOTROPY AND PRODUCT STATE DISTRIBUTION IN THE PHOTODISSOCIATION OF DEUTERIUM PEROXIDE
}

\author{
Karl-Heinz. GERICKE, Stefan KI.EE and Franz. Josef COMES \\ Institut für Physikalische und Theoretische Chemie der Johann Wolfgang Goethe-Universität, \\ Niederurseler Hang, D-6000 Frankfurt am Main 50, Federal Republic of Germany
}

Received 20 March 1987

\begin{abstract}
Nascent $\mathrm{OD}\left(\mathrm{X}^{2} \Pi_{1}\right)$ radicals, generated in the photodissociation of $\mathrm{D}_{2} \mathrm{O}_{2}$ at $266 \mathrm{~nm}$, have been probed by laser-induced fluorescence. The observed line intensities exhibit a marked dependence on excitation-detection geometry and laser polarisation, indicating rotational alignment $\left(A \delta^{2} \leqslant 0.28\right)$. The analysed product rotational distributions and Doppler width measurements indicate that the fragment energy partitioning $\left(f_{\mathrm{k} \text { nn }}=0.86, f_{\mathrm{rot}}=0.14\right)$ is too different from that reported for $\mathrm{H}_{2} \mathrm{O}_{2}$ photodissociation to explain the isotopic process by energy arguments alone.
\end{abstract}

\section{Introduction}

The photodissociation of hydrogen peroxide is an excellent example for the study of the fragmentation dynamics of polyatomic molecules. Angular momentum distributions and anisotropies of the recoiling fragments have been examined through analysis of the Doppler-broadened profiles of individual rotational transitions by laser-induced fluorescence [1-5]. Measurements of the correlation between $\boldsymbol{\mu}_{\mathrm{H}_{2} \mathrm{O}_{2}}$, the transition moment in the parent hydrogen peroxide, and $v_{\mathrm{OH}}$, the fragment translational motion, show that the first excited electronic state of $\mathrm{H}_{2} \mathrm{O}_{2}$ is of 'A symmetry [1] and the dissociation process at $266 \mathrm{~nm}$ can be characterised by

$$
\begin{aligned}
& \mathrm{H}_{2} \mathrm{O}_{2}\left(\tilde{\mathrm{X}}^{1} \mathrm{~A}\right)+h \nu(\lambda=266 \mathrm{~nm}) \rightarrow \mathrm{H}_{2} \mathrm{O}_{2}\left(\tilde{\mathrm{A}}^{1} \mathrm{~A}\right) \\
& \quad \rightarrow \mathrm{OH}\left(\mathrm{X}^{2} \Pi\right)+\mathrm{OH}\left(\mathrm{X}^{2} \Pi\right) .
\end{aligned}
$$

The vector correlation between the translational $\left(v_{\mathrm{OH}}\right)$ and rotational $\left(\mathrm{J}_{\mathrm{OH}}\right)$ moment of the photofragment in reaction (1) has been quantitatively established [2]. This correlation is positive and increases with increasing $J_{\mathrm{OH}}$ with a bias towards $v_{\mathrm{OH}}$ and $J_{\mathrm{OH}}$ being parallel to one another. Therefore, the internal torsional vibration in the peroxide molecule is obviously an important promoting mode for fragment rotational excitation in the dissociation pro- cess (1). The overall $\mathrm{OH}$ rotational distribution can be fitted to a Boltzmann distribution with a rotational temperature parameter of $T_{\text {rot }}=1500 \mathrm{~K}$. Only for low rotations is the population of the corresponding quantum states lower than predicted by the parameter $T_{\text {rot }}$. OH fragments generated in the photolysis of $\mathrm{H}_{2} \mathrm{O}_{2}$ at $248 \mathrm{~nm}$ show a similar rotational distribution [3]. However, on absorption of radiation at shorter wavelength $(193 \mathrm{~nm})$, a strongly inverted rotational state population for $\mathrm{OH}\left(\mathrm{X}^{2} \Pi\right.$, $\left.v^{\prime \prime}=0, J^{\prime \prime}\right)$ is found [5].

Without the appropriate information on the shape of the upper excited state potential surface, questions still remain as to the ultimate origin of the fragment rotation. The possibilities are that parent vibrational or rotational motion in the electronic ground state is carried through the dissociation process, or the torsional dependence of the transition to the excited state potential causes product internal excitation. Determining which factor contributes the most is the motivation of the present study.

Experiments which can determine the influence of the parent ground state distribution on that of the product state are those where the thermal energy content of the parent molecule is altered by cooling in a supersonic expansion or by changing the characteristic frequency through an isotope replacement. Supersonic beams provide a source of molecules with 
an extremely narrow velocity distribution. Such molecules are cooled to an extent that the excited rotational and vibrational levels of the molecules are almost completely depopulated. This greatly reduces the influence of thermal excitation of the parent molecule on the dynamics of the fragmentation. However, in the case of $\mathrm{H}_{2} \mathrm{O}_{2}$, e.g., the bending modes $\left(\nu_{4}, \nu_{6}\right)$ of the molecule remain "untouched", because even at room temperature the population of vibrationally excited levels is negligible.

The other means of studying the origin of hydroxyl fragment rotation is the replacement of light hydrogen atoms by heavier deuterium isotopes. This changes the vibrational and rotational frequencies in the parent molecule as well as the impulse lever-arm but keeps the electronic energy comparable to that of $\mathrm{H}_{2} \mathrm{O}_{2}$. If molecular motions involving the $\mathrm{H}$ atoms of hydrogen peroxide make a considerable contribution to the dissociation dynamics, then deuteration will result in a different rotational energy distribution and altered vector correlations.

In the present study, photofragmentation of $\mathrm{D}_{2} \mathrm{O}_{2}$ at $266 \mathrm{~nm}$ is discussed. The rotational state distribution of $\mathrm{OD}\left(\mathrm{X}^{2} \Pi_{1}, v^{\prime \prime}, J^{\prime \prime}\right)$ products and the vector correlation between $\mu_{\mathrm{D}_{2} \mathrm{O}_{2}}$ and $J_{\mathrm{OD}}$, described by the alignment parameter $A_{\delta}^{(2)}$, were obtained from observations of the fragment LIF intensity at different pump-probe geometries. In addition, Doppler profile measurements have been carried out to obtain detailed information on the velocity of the recoiling OD photoproducts.

\section{Experimental}

In order to determine the rovibronic population distribution and the rotational alignment of OD fragments we used the experimental apparatus already described elsewhere $[1,2]$. Deuterium peroxide was prepared by mixing $\mathrm{H}_{2} \mathrm{O}_{2}(90 \%)$ with an excess of $\mathrm{D}_{2} \mathrm{O}$ and distilling the mixture to higher peroxide concentration. This procedure is based on an isotope exchange reaction, which is known to be nearly instantaneous [6]. The deuterium peroxide obtained was of $90-95 \%$ chemical purity with regard to the measured refraction, and, moreover, the isotopic purity was found to be greater than $96 \%$ by comparing the $\mathrm{OD}$ and $\mathrm{OH}$ signal strengths under the assumption of similar parent absorbtivities. Since the absorption cross section of $\mathrm{D}_{2} \mathrm{O}_{2}$ in comparison to $\mathrm{H}_{2} \mathrm{O}_{2}$ is actually slightly reduced [7], the observed isotopic purity should be even higher.

$\mathrm{D}_{2} \mathrm{O}_{2}$ was rapidly pumped through a photolysis cell at pressures typical in the range 1-10 mTorr. Irradiation with the frequency-quadrupled output of a Nd:YAG laser $\left(\approx 10 \mathrm{MW} \mathrm{cm}^{-2}\right)$ yielded OD radicals, which were detected after a delay of $18 \mathrm{~ns}$ by laser-induced fluorescence in the $(0,0)$ band of the ${ }^{2} \Sigma^{+}-{ }^{2} \Pi_{3 / 2,1 / 2}$ transition. The probing dye laser $(\approx 1$ $\mathrm{kW} \mathrm{cm}^{-2}$ ) overlapped with the photodissociating laser beam in the middle of the cell. Each laser beam was linearly polarised, their planes of polarisation could be rotated by $\lambda / 2$ plates. The applied laser beam arrangement enabled measurements at both the mutually orthogonal and the counterpropagated coaxially probed geometry to be taken.

The undispersed fluorescence was always detected at right angles to the propagation direction of both the photolysing and analysing laser beams. Excitation spectra of $\mathrm{OD}\left(\mathrm{X}^{2} \Pi_{1}\right)$ were recorded between 306 and $312 \mathrm{~nm}$ at each of the eight possible photolysis-analysis detection geometries. The approximately 160 detected OD absorption lines for each spectrum were assigned on the basis of the spectroscopic work of Clyne et al. [8]. The OD transition probabilities used in the calculations were from Dimpfl and Kinsey [9].

In addition to the measurement of the absorption spectrum, which yields information concerning the partitioning of the available energy over the single rovibrational states with respect to their sublevels (spin and $\Lambda$ splitting), we determined the kinetic excitation of the ejected photofragments by recording high resolution excitation spectra of single rotational transitions.

\section{Results and discussion}

\subsection{Rotational alignment of $O D$ fragments}

Consideration of just the different lineshapes originating from $\mu-v$ and $v-J$ correlation effects by digitally integrating the line profiles is, in principle, not sufficient to deduce the proper internal state distributions. It is necessary to take into account the pos- 
sibility of fragment rotational anisotropy $(\mu-J$ correlation), which, if wrongly interpreted, may mislead one, as in the case of $\mathrm{X}^{2} \Pi_{1}$ product radicals to conclude an unequal population of the $\Lambda$ doublets. The theory of rotational anisotropy has been presented in a number of classic papers [10-13] and has been refined within the theoretical framework of vectorial properties of molecular motions, which was recently developed by Dixon [14]. There the possible correlations of photofragment translation and rotation are described. Experimentally observed laser-induced fluorescence is expressed in terms of simple geometrical factors and dynamical parameters $A_{\delta}^{(k)}$ corresponding to multipole expansion terms of the total angular momentum density matrix. In the case of photodissociation with linearly polarised light only two non-zero moments exist: the monopole moment $A \delta^{(0)}=1$ and the quadrupole moment $A_{0}^{(2)}$ ("alignment"). The latter vanishes for $J=0,1 / 2$ and lies between the limits

$-2 / 5 \leqslant A_{0}^{(2)} \leqslant 4 / 5$

for high rotational quantum numbers, corresponding to the limiting cases of fragment rotation $J$ perpendicular or parallel, respectively, to the parent molecule transition dipole $\mu$.

Rotational alignment can be determined by measuring the differences between relative line intensities as a function of different geometries and polarisation patterns in the photodissociation-detection laser configuration. If the LIF intensity turns out to be independent of the polarisation relations, then the spatial orientation of the molecular rotational vectors is isotropic. Otherwise, any change in the relative intensities indicate that rotational anisotropy is present and the associated moment $A_{\delta}^{(2)}$ is non-zero.

The intensity $I$ of a detected spectral line results from the individual rotational state population $P(J)$, the accompanying transition probability $B$, and the rotational alignment, where

$$
I \sim P(J) B \sum_{l, k} a_{\imath} A_{0}^{(k)} \text {. }
$$

The specific molecular coefficients $a_{l}(i=0,1)$ for OD were calculated using Dixon's theory [14]. These multipliers refer to the excitation-detection geometry and the angular momentum coupling. Hyperfine depolarisation as a result of nuclear precession as well

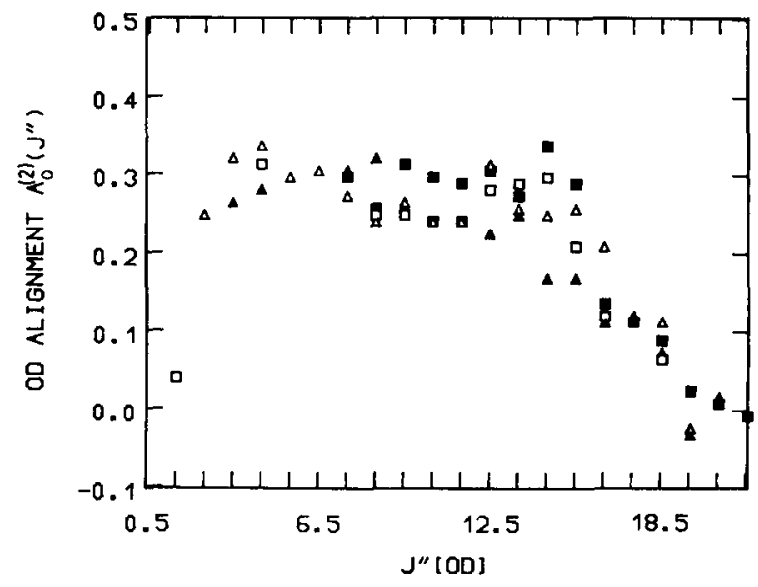

Fig. 1. The alignment parameter $A_{\delta}^{(2)}$ versus the angular momentum $J^{\prime \prime}$ of the OD photofragment: squares refer to the $\Pi_{3 / 2}$ and triangles to the $\Pi_{1 / 2}$ spin system, respectively. Filled symbols represent values belonging to the upper $A$ rotational state levels $\left(\Pi^{-}\right)$, open symbols label the lower $A$ levels $\left(\Pi^{+}\right)$.

as satellite contributions to the emitted fluorescence of $\mathrm{OD}\left({ }^{2} \Sigma^{+}\right)$main transitions have been considered.

The rotationally resolved photofragment alignment is extracted by determining the ratio of individual line intensities at each of two selected experimental polarisation geometries and forming average values of $A_{\delta}^{(2)}(J)$ for all spin- $A$ subsystems $\left(\Pi_{3 / 2}^{+}, \Pi_{\overline{3} / 2}, \Pi_{1 / 2}^{+} \Pi_{\overline{1 / 2}}\right)$. The result of this analysis for the OD alignment is shown in fig. 1: Apparently no regular variations exist between different sublevels greater than the experimental uncertainty of \pm 0.05 . The average alignment for $J_{\mathrm{OD}} \leqslant 15.5$ was found to be positive and nearly constant at $A \delta^{(2)}=$ 0.28 , whereas for higher $J_{\mathrm{OD}}$ alignment decreases reaching the limiting rotational level $J_{\mathrm{OD}}^{\max } \approx 22.5$ with $A \delta^{2)} \approx 0$.

Compared to the rotational alignment of $\mathrm{OH}$ radicals from the $266 \mathrm{~nm}$ photodissociation of $\mathrm{H}_{2} \mathrm{O}_{2}$ the shape of the $A_{\delta}^{(2)}(J)$ functions are similar, with the $J_{\mathrm{OD}}$ scale being expanded and the absolute value of the OD alignment at moderate rotational excitation being more than twice that of $\mathrm{OH}$ $\left(\left\langle A \delta^{2)}[\mathrm{OH}]\right\rangle \leqslant 0.1\right)[2]$. This would indicate that the OD rotational vector points more towards the direction of the parent molecule transition dipole, as in the case of the $\mathrm{OH}$ photoproduct. This significant difference in the dissociation dynamics of isotopic molecules may be caused by different weightings of 
the parent bending and torsion vibrational motions when they are transformed into the diatomic fragment rotational distribution during the dissociation process.

\subsection{OD internal state distribution}

Since significant alignment occurs in the photofragmentation process, the single spectral line intensities must be corrected according to eq. (3) to obtain reliable population numbers. The correction accounts for as much as $30 \%$ of the raw intensity depending on the rotational excitation and the spectroscopic transition chosen. Fig. 2 shows the resulting rotational state distribution of the vibrationless levels of OD for the ${ }^{2} \Pi_{3 / 2}$ and ${ }^{2} \Pi_{1 / 2}$ states. The population of a particular rotational state is given as the arithmetic mean of the $\Pi^{+}$and $\Pi^{-}$components. The rotational distribution demonstrates that (i) the dissociation process does not distinguish between the different spin states, (ii) the most probable rotational excitation occurs at $N_{\mathrm{OD}}=11$ in contrast to the isotopic photofragmentation of $\mathrm{H}_{2} \mathrm{O}_{2}$, where the distribution peaks at $N_{\mathrm{OH}}=6$ [2] and (iii) the rotational excitation function has a Gaussian and not a Boltzmann distribution and hence is not describable by a rotational temperature parameter. Fig. 2 shows the spinlevel-resolved rotational state distribution, which was fitted with a Gaussian function of the form

$P\left(N_{\mathrm{OD}}\right) \sim \exp \left[-4 \ln 2\left(N_{\mathrm{OD}}-\bar{N}_{\mathrm{OD}}\right)^{2} / \Delta N_{\mathrm{OD}}^{2}\right]$

and which peaks at $\bar{N}_{\mathrm{OD}} \approx 11$ with a width (fwhm) of $\Delta N_{\mathrm{OD}} \approx 9$.

There are a number of rotational state distributions for the dissociation of simple polyatomic molecules reported in the literature which qualitatively look like that obtained in the present experiment. These usually smooth and inverted excitation functions are in agreement with the angular reflection principle for scattering and photodissociation processes [15], which mediates between the parent bound state wavefunction, the anisotropy of the repulsive potential, and the fragment final state distribution.

It is found in our experiment that the OD fragments are preferentially formed in the upped $\Pi^{-} \Lambda$ component. This feature was already observed in the $266 \mathrm{~nm}$ photodissociation process of $\mathrm{H}_{2} \mathrm{O}_{2}$. Sum-

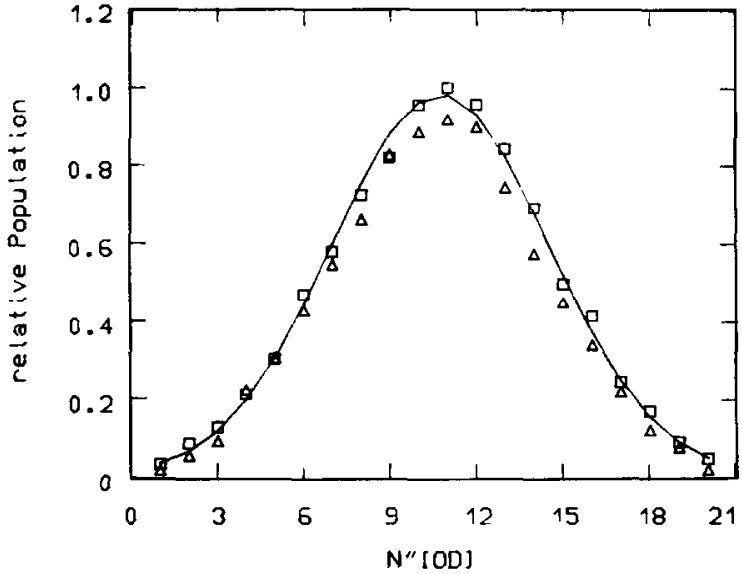

Fig. 2. OD rotational state populations of the ${ }^{2} \Pi_{3 / 2}$ (squares) and ${ }^{2} \Pi_{1 / 2}$ (triangles) spin states after dissociation of $D_{2} \mathrm{O}_{2}$ at $266 \mathrm{~nm}$. The population has been corrected for the effect of rotational alignment (see text). The solid line represents a Gaussian fit to the population numbers, obtained by a least-squares-fit procedure.

ming over all accessible OD rotational states within a $A$ subsystem yields a population fraction of $P\left(\Pi^{-}\right): P\left(\Pi^{+}\right)=0.55: 0.45$. The rotational dependence of this population inversion as well as the associated dynamical implications will be discussed in a forthcoming paper [16] in connection with evaluated vector correlations between product rotational and translational motions along with parent dissociative conformation and transition moment orientation.

Vibrationally excited OD products were not observed. From the signal-to-noise ratio and the transitions belonging to $\mathrm{X}^{2} \Pi_{1}, v^{\prime \prime}=0$ we determined an upper limit for OD formed in $v^{\prime \prime}=1$ to be less than $2 \%$, which is a negligible amount in the overall mechanism of the photodissociation process in the long-wavelength region of the hydrogen peroxide UV absorption.

\subsection{Energy partitioning}

The total excess energy $E_{\text {av }}$, transferred during the photofragmentation process into the products, is determined by the dissociation energy $E_{\mathrm{d}}$, the photon energy of the photolysing laser $h \nu$, and the initial energy of the parent molecule $E_{\text {in }}$ :

$E_{\mathrm{av}}=h \nu+E_{\mathrm{in}}-E_{\mathrm{d}}$. 
Our estimate of $E_{\mathrm{d}}\left(\mathrm{D}_{2} \mathrm{O}_{2}\right)$ is based on the precisely known dissociation energy of hydrogen peroxide, $E_{\mathrm{d}}\left(\mathrm{H}_{2} \mathrm{O}_{2}\right)=207 \mathrm{~kJ} \mathrm{~mol}^{-1}$ [17], and on calculating the influence of isotopic substitution with regard to the zero-point energies in the peroxide molecules as well as in the hydroxyl products. The zero-point energies in the parent molecules are lowered by $\Delta E_{0}\left(\mathrm{R}_{2} \mathrm{O}_{2}\right)=15.7 \mathrm{~kJ} \mathrm{~mol}^{-1}$, whereas for both $\mathrm{OD}$ fragments the energy is reduced by $2 \Delta E_{0}(\mathrm{OR})=12.1$ $\mathrm{kJ} \mathrm{mol}^{-1}$. Derived from this is an overall energy difference of $3.6 \mathrm{~kJ} \mathrm{~mol}^{-1}$ for $\mathrm{O}-\mathrm{O}$ cleavage in isotopic processes, which is in agreement with the experimentally observed hypsochromic shift of the $\mathrm{D}_{2} \mathrm{O}_{2}$ absorption continuum relative to that of $\mathrm{H}_{2} \mathrm{O}_{2}$ [7]. Hence the dissociation energy of $\mathrm{D}_{2} \mathrm{O}_{2}$ amounts to $211 \mathrm{~kJ} \mathrm{~mol}^{-1}$.

At room temperature, the parent deuterium peroxide molecules have an average rotational energy of $E_{\mathrm{r}}=\frac{3}{2} R T$ and a smaller amount of vibrational energy, $E_{v}$, mainly originating from the $\nu_{4}$ torsional mode:

$$
\begin{aligned}
& E_{1 \mathrm{n}}\left(\mathrm{D}_{2} \mathrm{O}_{2}\right)=E_{\mathrm{v}}\left(\mathrm{D}_{2} \mathrm{O}_{2}\right)+E_{\mathrm{r}}\left(\mathrm{D}_{2} \mathrm{O}_{2}\right) \\
& =5.2 \mathrm{~kJ} \mathrm{~mol}^{-1} .
\end{aligned}
$$

As the consequence of zero-point lowering and the smaller vibrational level spacing, the initial internal energy of $\mathrm{D}_{2} \mathrm{O}_{2}$ exceeds that of $\mathrm{H}_{2} \mathrm{O}_{2}$ by $8 \%$. The total energy available for excitation of the two OD fragments is $\left(h \nu=449.7 \mathrm{~kJ} \mathrm{~mol}^{-1}\right)$

$E_{\mathrm{av}}=244 \mathrm{~kJ} \mathrm{~mol}^{-1}$.

Most of this energy is released into OD translation, which was experimentally confirmed by measuring the Doppler shift of the recoiling photofragments. Fig. 3 shows high resolution spectra of the $R_{4}(4)$ and $Q_{1}(18)$ transitions of $O D\left(X^{2} \Pi_{1}\right)$. In order to obtain the Doppler width of these profiles one has to deconvolute the obtained lineshapes from a Gaussian function which reflects the influence of the $300 \mathrm{~K}$ thermal translational distribution of $\mathrm{D}_{2} \mathrm{O}_{2}$ and the finite dye laser resolution [1]. The value for the Doppler shift was found to depend markedly on the fragment rotational excitation: the $J=3.5$ rotational line has a width ( $\mathrm{fwhm}$ ) of $\Delta \nu_{\mathrm{D}}=0.392 \pm 0.020 \mathrm{~cm}^{-1}$, which corresponds to an $O D$ recoil velocity of $v_{\mathrm{OD}}=3610 \pm 180 \mathrm{~m} \mathrm{~s}^{-1}$ and the $J=18.5$ transition yields $\Delta \nu_{\mathrm{D}}=0.353 \pm 0.020 \mathrm{~cm}^{-1}$ corresponding to $\nu_{\mathrm{OD}}=3280 \pm 190 \mathrm{~m} \mathrm{~s}^{-1}$.

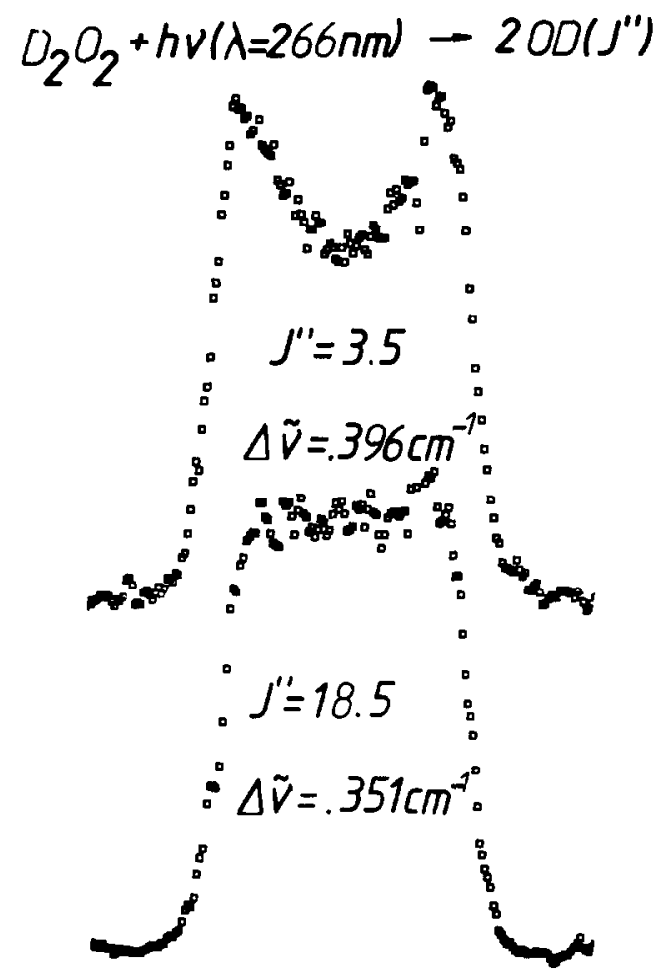

Fig. 3. High-resolution $O D$ excitation spectra of the $R_{2}(4)$ $(J=3.5)$ and $Q_{1}(18)(J=18.5)$ rotational transitions. The inserted values for Doppler widths, $\Delta \nu\left(J^{\prime \prime}\right)$, have been obtained considering the $300 \mathrm{~K}$ thermal motion of $\mathrm{D}_{2} \mathrm{O}_{2}$ and the Gaussian laser profile. The striking difference between the lineshapes shown is a consequence of $v_{\mathrm{OD}}-J_{\mathrm{OD}}$ correlation.

The precision of the Doppler profile measurements allows a determination of the fragment translational excitation by summing up the individual kinetic energy fraction of each populated rotational state. This procedure is, if vibrational excitation is absent, a more direct one in comparison with determinations based on the conservation of energy according to

$\left\langle E_{\mathrm{kin}}\right\rangle=E_{\mathrm{av}}-\left\langle E_{\mathrm{rot}}\right\rangle-\left\langle E_{\mathrm{vib}}\right\rangle$.

The value resulting from Doppler width analysis, $\left\langle E_{\text {trans }}\right\rangle=214 \mathrm{~kJ} \mathrm{~mol}^{-1}$, agrees well within $2 \%$ with that calculated on the basis of the energy conservation principle (8), assuming no vibrationally excited OD product.

A summary of energy partitioning in the $266 \mathrm{~nm}$ photodissociation of deuterium peroxide in comparison to the corresponding observations in the 
Table 1

Comprehensive view over the energy partitioning (energies in $\mathrm{kJ} \mathrm{mol}^{-1}$ ) in the $266 \mathrm{~nm}$ photodissociation, $\mathrm{R}_{2} \mathrm{O}_{2}+h \nu \rightarrow 2 \mathrm{OR}\left(\mathrm{X}^{2} \Pi_{1}\right)$, of deuterium peroxide (present work), hydrogen peroxide [2], and hydrogen peroxide at the shorter wavelengths of 248 [3] and $193 \mathrm{~nm}$ [5]. The OD product kinetic excitation has been measured by Doppler profile analysis, whereas that of $\mathrm{OH}$ has been calculated in each case according to the energy conservation principle (see text)

\begin{tabular}{llllllllll}
\hline $\mathrm{R}$ & $\lambda(\mathrm{nm})$ & $E_{\mathrm{av}}$ & $E_{\text {diss }}$ & $E_{\mathrm{ln}}$ & $\left\langle E_{\mathrm{kin}}\right\rangle$ & $\left\langle E_{\text {ror }}\right\rangle$ & $f_{\text {kin }}$ & $f_{\text {ror }}$ & Ref. \\
\hline $\mathrm{D}$ & 266 & 244 & 211 & 5.2 & 214 & 33 & 0.87 & 0.13 & this work \\
$\mathrm{H}$ & 266 & 248 & 207 & 4.8 & 223 & 25 & 0.90 & 0.10 & {$[2]$} \\
$\mathrm{H}$ & 248 & 280 & 207 & 4.8 & 250 & 30 & 0.89 & 0.11 & {$[3]$} \\
$\mathrm{H}$ & 193 & 417 & 207 & 4.8 & 350 & 67 & 0.84 & 0.16 & {$[5]$} \\
\hline
\end{tabular}

fragmentation of hydrogen peroxide at 266,248 , and $193 \mathrm{~nm}$ is given in table 1 . This energy partitioning into the various degrees of freedom for $\mathrm{D}_{2} \mathrm{O}_{2}$ and $\mathrm{H}_{2} \mathrm{O}_{2}$ photodissociation at $266 \mathrm{~nm}$ reveals that the OD fragment receives $\approx 30 \%$ more rotational excitation than the isotopic $\mathrm{OH}$, although $E_{\mathrm{av}}\left(\mathrm{D}_{2} \mathrm{O}_{2}\right) \approx E_{\mathrm{av}}\left(\mathrm{H}_{2} \mathrm{O}_{2}\right)$. This tendency becomes more marked at $248 \mathrm{~nm}$. In the latter case the products have $\pm 15 \%$ more energy at their disposal, but the outcome is $\approx 10 \%$ less rotational excitation. Only at still shorter photolysis wavelengths of, e.g., 193 $\mathrm{nm}$ does the fragment rotational excitation increases, but now contributions from a second excited potential surface $\left(\widetilde{\mathrm{B}}^{1} \mathrm{~B}\right)$ are present [18]. Consequently, the difference in product internal energy disposal in the course of $266 \mathrm{~nm}$ photolysis of the isotopic peroxides is too pronounced to be explained by energy considerations alone. Further information on the photodissociation dynamics is needed. This may be gained from a complete analysis of molecular vectorial properties, such as $\boldsymbol{\mu}-\boldsymbol{v}$ and $\boldsymbol{v}-\boldsymbol{J}$ correlation as well as from that between the rotational angular momenta (including the different spin and $\boldsymbol{A}$ substates) of both partner fragments, generated in the same elementary process.

\section{Acknowledgement}

We thank Professor R.N. Dixon (Bristol) for making available his calculations of the multipliers of the OD bipolar moments. The work has been performed as part of a program of the Deutsche For- schungsgemeinschaft, whose financial support is gratefully acknowledged.

\section{References}

[1] S. Klee, K.-H. Gericke and F.J. Comes, J. Chem. Phys. 85 (1986) 40.

[2] K.-H. Gericke, S. Klee, F.J. Comes and R.N. Dixon, J. Chem. Phys. 85 (1986) 4463.

[3] M.P. Docker, A. Hodgson and J.P. Simons, Chem. Phys. Letters 128 (1986) 264.

[4] M.P. Docker, A. Hodgson and J.P. Simons, Faraday Discussions Chem. Soc., to be published.

[5] A.U. Grunewald, K.-H. Gericke and F.J. Comes, Chem. Phys. Letters 132 (1986) 121.

[6] H. Erlenmeyer and H. Gaertner, Helv. Chim. Acta 17 (1934) 970.

[7] M.K. Phibbs and P.A. Giguere, Can. J. Chem. 29 (1951) 490.

[8] M.A.A. Clyne, J.A. Coxon and A.R. Woon Fat, J. Mol. Spectry. 46 (1973) 146.

[9] W.L. Dimpfl and J.K. Kinsey, J. Quant. Spectry. Radiative Transfer 21 (1979) 233.

[10] U. Fano and J.H. Macek, Rev. Mod. Phys. 45 (1973) 553.

[11] D.A. Case, G.M. McClelland and D.R. Herschbach, Mol. Phys. 35 (1978) 541.

[12] C.H. Greene and R.N. Zare, Ann. Rev. Phys. Chem. 33 (1982) 119.

[13] C.H. Greene and R.N. Zare, J. Chem. Phys. 78 (1983) 6741.

[14] R.N. Dixon, J. Chem. Phys. 85 (1986) 1866.

[15] R. Schinke, J. Phys. Chem. 90 (1986) 1742; J. Chem. Phys. 85 (1986) 5049.

[16] S. Klee, K.-H. Gericke and F.J. Comes, to be published.

[17] H. Okabe, Photochemistry of small molecules (Wiley, New York, 1978), p. 282.

[18] A.U. Grunewald, K.-H. Gericke and F.J. Comes, to be published. 